\title{
Pain and efficacy of local anesthetics for central venous access
}

\author{
William C Culp Jr' \\ Mohammed Yousaf ${ }^{2}$ \\ Benjamin Lowry' \\ Timothy C McCowan ${ }^{3}$ \\ William C Culp ${ }^{2}$ \\ 'Division of Cardiothoracic \\ Anesthesiology, Scott and White \\ Hospital, The Texas A\&M University \\ College of Medicine, Temple, TX, USA; \\ ${ }^{2}$ Division of Interventional Radiology, \\ University of Arkansas for Medical \\ Sciences, Little Rock, AR, USA; \\ ${ }^{3}$ Department of Radiology, University \\ of Mississippi Medical Center. \\ Jackson, MS, USA
}

Purpose: To compare pain during injection and efficacy of analgesia of local anesthetics during central venous line placement.

Methods: Sixty-two patients were studied in a randomized, double-blinded prospective fashion. Patients received $1 \%$ lidocaine (L), buffered $1 \%$ lidocaine (LB), or $2 \%$ chloroprocaine (CP) injected around the internal jugular vein for procedural analgesia for central venous access. Patients reported pain via a standard linear visual analog scale, with 0 representing no pain and 10 being the worst pain imaginable.

Results: Overall patient perception of pain was better with $\mathrm{CP}$ and $\mathrm{L}$ than $\mathrm{LB}$ with mean scores of CP 2.4, L 2.6, LB 4.2. Pain with injection mean scores were CP 2.1, L 2.5, LB 3.2. Pain with catheter placement scores were CP 2.5, L 1.7, LB 3.4. Operator assessment of overall pain values were CP 1.9, L 2.2, LB 3.4. LB consistently scored the worst, though compared with $\mathrm{CP}$, this only reached statistical significance in overall patient pain and pain at catheter insertion compared with L.

Conclusion: Though chloroprocaine scored better than lidocaine in 3 of 4 parameters, this trend did not achieve statistical significance. Adding sodium bicarbonate to lidocaine isn't justified in routine practice, nor is routine replacement of lidocaine with chloroprocaine.

Keywords: local anesthesia, analgesia, central venous access, lidocaine, chloroprocaine

\section{Introduction}

Central venous catheter placement as well as many other surgical procedures can be painful, and this pain is often reduced with the use of local anesthetics. The local anesthetic used to reduce pain, however, often causes pain itself. The best local anesthetic compound to utilize in order to minimize this pain upon injection while still providing adequate and effective anesthesia for various procedures has long been the center of controversy. Several studies have suggested that a buffered lidocaine (LB) solution causes less pain during intra- and subdermal injection in comparison with the most commonly used local anesthetic, lidocaine (L) (Nuttal et al 1993; Prien 1994; Xia et al 2002; Davies 2003). Alternatively, other data suggest that chloroprocaine (CP) is associated with the least amount of pain on injection and/or that buffering $\mathrm{L}$ does not decrease pain on injection (Marica et al 2002; Burns et al 2006). This study attempts to assess the analgesic efficacy and associated pain on injection of three local anesthetic solutions used for procedural analgesia for central venous access.

\section{Methods}

After approval of the local Institutional Review Board, 62 consecutive patients requiring central venous access with planned placement in the internal jugular vein were consented and enrolled in the study. Each patient was randomized and assigned into a group via computer randomizer program to receive one of three local anesthetics for procedural analgesia. Each patient was to receive $5 \mathrm{~mL}$ of either L 1\% (Xylocaine 1\%, 
Astra Zeneca LP, Wilmington, DE, USA), alkalinized L 1\% prepared with $8.4 \%$ sodium bicarbonate in a $1: 10$ ratio (LB) (Xylocaine 1\%, Astra Zeneca LP; sodium bicarbonate 8.4\%, American Regent, Inc., Shirley, NY, USA), or 2\% CP (Nesacaine 2\%, Bedford Laboratories, Bedford, OH, USA). These medicines were prepared at room temperature 30 minutes prior to the procedure by a research pharmacist. Twenty minutes prior to the procedure, each patient received diazepam $5 \mathrm{mg}$ by mouth for mild anxiolysis. No additional sedatives or analgesics were administered. As the patient was brought into the procedure room, the operator was provided with a syringe containing one of the randomly assigned local anesthetics. After confirming suitability of the target jugular vein by ultrasound, the operator then injected $3 \mathrm{~mL}$ of the local anesthetic solution through a 25 gauge needle directly superficial to the internal jugular vein with ultrasound guidance. This injection was deliberate and not rushed, lasting $5-10 \mathrm{sec}$. The needle was then repositioned to inject $1 \mathrm{~mL}$ just to the left and $1 \mathrm{~mL}$ just to the right of the vein for anchoring stitches (Figure 1). Each patient received a 7 Fr triple-lumen catheter via a nontunneled approach.

Each patient also underwent instruction on rating pain via a standardized $100 \mathrm{~mm}$ horizontal linear visual analog pain scale in which a score of 0 represented no pain and 10 represented the worst possible pain (Katz and Melzack 1999). Then, pain scores were reported by the patient to a nurse observer after initial local anesthetic injection, after central line insertion, and after the overall procedure was completed. Additionally, the operator reported his assessment of overall procedural patient pain. The operator and nurse observer were blinded to each other's recorded pain scores. All participants in the study (patients, nurse observers, and operator) were blinded to the study drugs until after statistical analysis was complete.

Analysis of variance was used to examine the distribution of pain score responses across each of the conditions of interest. ANOVA and Kruskal-Wallis nonparametic tests were applied for comparisons of drugs. The mean pain scores were reported with the standard error of the mean. Statistical significance was defined as a probability value of $<0.05$.

\section{Results}

Sixty-two patients were enrolled, with all but 2 completing the study. One was excluded due to a lack of a patent jugular vein and one was excluded after the operator opted to cannulate the subclavian vein instead of the internal jugular vein.

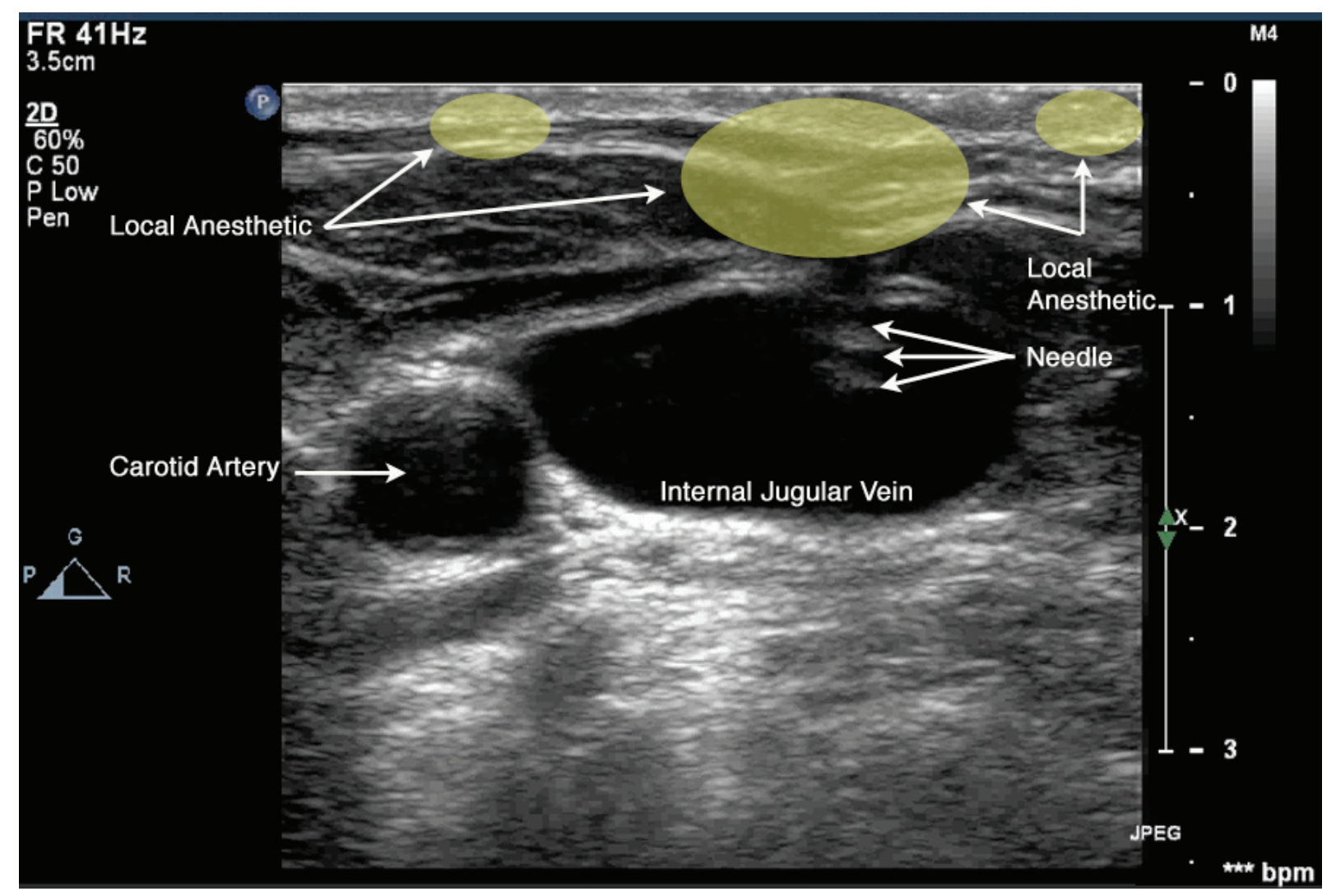

Figure I This two-dimensional ultrasound image depicts the internal jugular vein and the target areas for infiltration of local anesthetic solutions. 
There were no significant differences among the three randomized groups of patients in terms of age, gender, or co-morbidities (Table 1). Chronic opiate therapy was noted in 8 of 62 patients. The mean pain scores during injection, catheter placement, overall pain, and operator assessed score are depicted in Figure 2. Mean values $( \pm S D)$ are listed in Table 2. CP and L scored better than LB in overall patient perception of pain with means of CP 2.4, L 2.6, and LB 4.2 ( $p=0.01$ and 0.02 with $\mathrm{CP}$ and $\mathrm{L}$ versus $\mathrm{LB}$ respectively). $\mathrm{CP}$ versus $\mathrm{LB}$ failed to show statistical significance with a p-value of 0.08 . Mean scores of pain with placement of the catheter were CP 2.5, L 1.7, and LB 3.4. Again, CP versus LB showed no statistically significant difference, however, $L$ versus LB met statistical significance with a p-value of 0.02 . Operator assessment of overall pain scores were CP 1.9, L 2.2, and LB 3.4 and only one relationship was significant ( $p=0.09 \mathrm{CP}$ versus LB). LB mean scores were consistently the worst in each category, $\mathrm{p}=0.04$. Overall, $\mathrm{CP}$ and $\mathrm{L}$ were not statistically different with any parameter, though $\mathrm{CP}$ trended towards less pain in three of four parameters.

\section{Discussion}

Alleviating and avoiding procedural pain not only decreases anxiety and fear in patients, but it also facilitates performing the procedure and improves overall patient satisfaction. Multiple surveys and studies have shown improved patient satisfaction with overall surgical and anesthetic experience when perceived pain is less than expected (Myles et al 2000). Procedures utilizing local anesthetics as a primary analgesic have increased dramatically over the past few decades. Local anesthetics are utilized in a wide array of procedures ranging from facilitating placement of simple peripheral intravenous catheters to cardiac catheterization, complex interventional radiological procedures, and minimally-invasive surgical procedures (Tiwari et al 2006; Baril et al 2007).

Table I Subject demographics

\begin{tabular}{llll}
\hline Parameter & Lidocaine & $\begin{array}{l}\text { Buffered } \\
\text { lidocaine }\end{array}$ & Chloroprocaine \\
\hline $\begin{array}{l}\text { Number } \\
\text { of subjects }\end{array}$ & 20 & 19 & 21 \\
$\begin{array}{l}\text { Male gender } n(\%) \\
\text { Mean age (years) }\end{array}$ & $12(60 \%)$ & II (58\%) & $14(67 \%)$ \\
$\begin{array}{l}\text { Subjects receiving } \\
\text { chronic opiate }\end{array}$ & $\mathrm{I}$ & 55.4 & 54.3 \\
\begin{tabular}{l} 
analgesics $(\mathrm{n})$ \\
\hline
\end{tabular}
\end{tabular}

The goal of utilizing local anesthetics is to decrease the pain associated with the operative procedure. Unfortunately, injection of the local anesthetics to reduce procedural pain is typically painful in itself. The precise mechanism of pain produced by intradermally or subcutaneously injected local anesthetics remains unclear. The $\mathrm{pH}, \mathrm{pKa}$, lipid solubility, temperature, concentration, technique of injection, site of injection, doctor-patient communication, pre-conceived expectations, or a combination of these or other characteristics have been suggested as possible factors affecting pain during injection (Morris and Whish 1984; McKay et al 1987; Nuttal et al 1993; Colaric et al 2000; Burns et al 2006; Tiwari et al 2006; Dutt-Gupta et al 2007; Leff et al 2007).

Most local anesthetics are produced in acidic solutions, with $\mathrm{pH}$ typically ranging from $5-7$. If the $\mathrm{pH}$ is increased towards the anesthetic's pKa by adding a buffer, the anesthetic may become less stable and more subject to photodegradation, denaturing reactions, and possible precipitation (Milner et al 2000; Davies 2003). Many physicians have advocated buffering local anesthetics with sodium bicarbonate just before administration thereby increasing the fraction of the nonionized base form, speeding the onset action, and decreasing associated pain with injection (Davies 2003). However, $\mathrm{pH}$ alone has been shown not to be an independent predictor of pain during injection (Marica et al 2002). In fact, the more acidic $\mathrm{CP}$ ( $\mathrm{pH} 3-4)$ has been shown to cause less pain upon intradermal injection than buffered L solutions ( $\mathrm{pH} 7-8)$. No difference in pain upon injection has been shown between $\mathrm{CP}(\mathrm{pH}$ 3-4) and buffered CP ( $\mathrm{pH} 7-8)$. Furthermore, sodium bicarbonate alone $(\mathrm{pH} 7-8.5)$ is more painful upon injection than many local anesthetics (Marica et al 2002). These data do not support the assertion that alkinilization of local anesthetics is the mechanism by which pain on injection is reduced with the addition of sodium bicarbonate.

These contradictory results appear to be related to study design and the vagaries of pain assessment, utilization of nonequivalent local anesthetic concentrations, differing additives and their concentrations, or different injection sites. Different patterns of nerve fibers in differing sites of injection may be a large source of these contradictory data, including our unexpected finding of relatively poor performance of LB. Our study called for injections as deep as $2-3 \mathrm{~cm}$ in the neck, in contrast to intradermal and immediate subdermal injections, most often in the hand or arm, as examined in most other similar studies. Since the precise mechanism of pain during injection of local anesthetics remains unclear, it is hard to predict which anesthetic will cause the most pain and as a result, clinical efficacy studies are the best means 
Local Anesthetic Mean Pain Scores

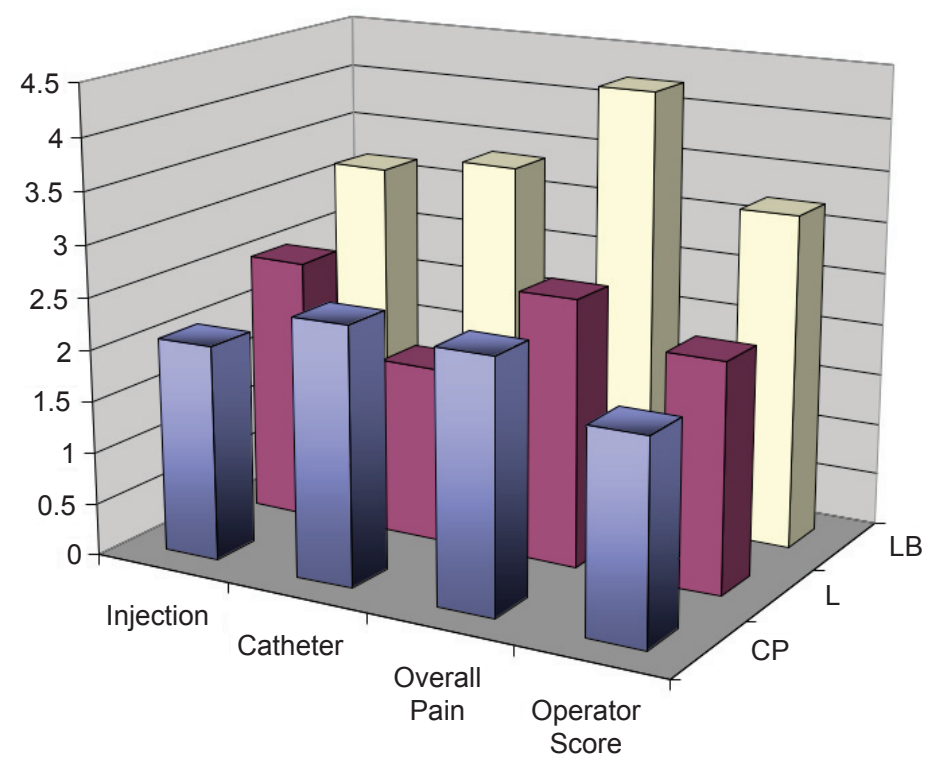

Figure 2 Mean pain scores with varying local anesthetic solutions as reported by the patients during initial injection, during catheter placement, overall pain, and pain as assessed by the operator.

of assessing these differences in anesthetic compounds in differing clinical applications.

This study attempts to do just this, and by using randomization, educating patients regarding reporting pain, strict blinding of all involved, multiple independent assessors of pain, and a reproducible stimulus (strictly guided by ultrasound), we sought to minimize study design induced variability in our data. There was some use of chronic opiate therapy in a small number of the patients (eight of 62 patients), which may potentially confound our results, although the mechanisms of analgesia via local anesthetics and opiates are clearly distinct. One other potential study weakness is the failure to detect a statistically significant difference in $\mathrm{CP}$ and $\mathrm{L}$ pain scores. By increasing sample size, future studies may be powered to prove a difference, if one exists. We believe that this study demonstrates that any difference between $\mathrm{CP}$ and $\mathrm{L}$ is too small to be clinically significant.

From a practical standpoint, the choice of local anesthetic for central venous access procedures is nearly always $\mathrm{L}$ due to

Table 2 Mean $( \pm S D)$ pain score results

\begin{tabular}{lllll}
\hline Agent & Injection & $\begin{array}{l}\text { Catheter } \\
\text { placement }\end{array}$ & Overall & $\begin{array}{l}\text { Operator } \\
\text { assessment }\end{array}$ \\
\hline Lidocaine & $2.5 \pm 1.8$ & $1.7 \pm 2.0$ & $2.6 \pm 2.0$ & $2.2 \pm 1.7$ \\
$\begin{array}{l}\text { Buffered } \\
\text { lidocaine }\end{array}$ & $3.2 \pm 2.4$ & $3.4 \pm 2.7$ & $4.2 \pm 2.7$ & $3.4 \pm 2.7$ \\
Chloroprocaine & $2.1 \pm 1.8$ & $2.5 \pm 2.1$ & $2.4 \pm 1.7$ & $1.9 \pm 1.6$ \\
\hline
\end{tabular}

tradition, its low cost and universal availability. Adding sodium bicarbonate immediately before injection takes additional time and adds $20 \%-30 \%$ to the cost of the local anesthetic mixture, while $\mathrm{CP}$ is roughly $4-5$ times more expensive than equal volumes of L (Cardinal Health, Dublin, OH, USA). Additionally, $\mathrm{CP}$ is an amino-ester local anesthetic, and as such, contains a para-aminobenzoic acid ring which is potentially allergenic. Therefore, the evidence of increased efficacy and/or less pain during injection should be compelling before recommending an alternative agent over $\mathrm{L}$ for routine use.

Our study demonstrated that for central venous access procedures, LB was associated with more procedural pain and pain on injection than L and CP. Though CP generally trended towards lower pain scores than $\mathrm{L}$, these failed to reach statistical significance. Based on these data, it is not justified to substitute $\mathrm{CP}$ for $\mathrm{L}$ in routine practice, nor is adding sodium bicarbonate to $L$ for central venous access procedures.

\section{Disclosure}

The authors report no conflicts of interest in this work.

\section{References}

Baril DT, Kahn RA, Ellozy SH, et al. 2007. Endovascular abdominal aortic aneurysm repair: emerging developments and anesthetic considerations. $J$ Cardiothorac Vasc Anesth, 21:730-42.

Burns CA, Ferris G, Feng C, et al. 2006. Decreasing the pain of local anesthesia: a prospective double-blind comparison of buffered, premixed $1 \%$ lidocaine with epinephrine versus $1 \%$ lidocaine freshly mixed with epinephrine. J Am Acad Dermatol, 54:128-31. 
Colaric KB, Overton DT, Moore K. 2000. Pain reduction in lidocaine administration through buffering and warming. Am J Emerg Med, 18:235-6.

Davies RJ. Buffering the pain of local anaesthetics: a systematic review. 2003. Emerg Med (Freemantle), 15:81-8.

Dutt-Gupta J, Brown T, Cyna AM. 2007. Effect of communication on pain during intravenous cannulation: a randomized controlled trial. $\mathrm{Br} J$ Anaesth, 99:871-5.

Katz J, Melzack R. 1999. Measurement of pain. Surg Clin North Am, 79:231-52.

Leff DR, Nortley M, Dang V, et al. 2007. The effect of local cooling on pain perception during infiltration of local anesthetic agents: a prospective randomized controlled trial. Anesthesia, 62:677-82.

Marica LS, O'Day T, Janosky JE, et al. 2002. Chloroprocaine is less painful than lidocaine for skin infiltration anesthesia. Anesth Analg, 94:351-4.

McKay W, Morris R, Mushlin P. 1987. Sodium bicarbonate attenuates pain on skin infiltration with lidocaine with or without epinephrine. Anesth Analg, 66:572-4.
Milner QJ, Guard BC, Allen JG. 2000. Alkalinization of amide local anesthetics by addition of $1 \%$ sodium bicarbonate solution. Eur $J$ Aneasthesiol, 17:38-42.

Morris RW, Whish DK. 1984. A controlled trial of pain on skin infiltration with local anesthetics. Anaesth Intensive Care, 12:113-4.

Myles PS, Williams DL, Anderson H, et al. 2000. Patient satisfaction after anesthesia and surgery: results of a prospective survey of 10,811 patients. BrJ Anaesth, 84:6-10.

Nuttall GA, Barnett MR. 1993. Establishing intravenous access: a study of local anesthetic efficacy. Anesth Analg, 77:950-3.

Prien TH. 1994. Intradermal anaesthesia: comparison of several compounds. Acta Anaesthesiol Scand, 38:805-7.

Tiwari A, Chawla R, Kumar M. 2006. Analgesia for venous cannulation by subcutaneous infiltration: effect of lidocaine concentration. Eur $J$ Anaesthesiol, 23:1063-4.

Xia Y, Chen E, Tibbits DL, et al. 2002. Comparison of effects of lidocaine hydrochloride, buffered lidocaine, diphenhydramine, and normal saline after intradermal injection. J Clin Anesth, 14:339-42. 
